\title{
Optically controlled polariton condensate molecules
}

\author{
E. D. Cherotchenko, ${ }^{1}$ H. Sigurdsson, ${ }^{2}$ A. Askitopoulos, ${ }^{3}$ and A. V. Nalitov ${ }^{4,5,6}$ \\ ${ }^{1}$ Ioffe Physical-Technical Institute of the Russian Academy of Sciences, St. Petersburg 194021, Russia \\ ${ }^{2}$ School of Physics and Astronomy, University of Southampton, Southampton SO17 1BJ, United Kingdom \\ ${ }^{3}$ Skolkovo Institute of Science and Technology, Bolshoy Boulevard 30, Building 1, Moscow, 121205, Russia \\ ${ }^{4}$ ITMO University, St. Petersburg 197101, Russia \\ ${ }^{5}$ Faculty of Science and Engineering, University of Wolverhampton, \\ Wulfruna Street, Wolverhampton WV1 1LY, UK \\ ${ }^{6}$ Institut Pascal, PHOTON-N2, Université Clermont Auvergne, 63001 Clermont-Ferrand, France
}

(Dated: March 27, 2021)

\begin{abstract}
A condensed-matter platform for analog simulation of complex two-dimensional molecular bonding configurations, based on optically trapped exciton-polariton condensates is proposed. The stable occupation of polariton condensates in the excited states of their optically configurable potential traps permits emulation of excited atomic orbitals. A classical mean-field model describing the dissipative coupling mechanism between $p$-orbital condensates is derived, identifying lowest-threshold condensation solutions as a function of trap parameters corresponding to bound and antibound $\pi$ and $\sigma$ bonding configurations, similar to those in quantum chemistry.
\end{abstract}

\section{INTRODUCTION}

The idea of designing analog simulators to study complex molecular systems relies on the ability to map such systems onto experimentally accessible and transparent platforms. Such simulators operate on the principle that a specially designed system can simulate interactions between artificial atomic or molecular orbitals through its parallelized and (ideally) fast dynamics. From this perspective, implementations of interacting artificial atoms - forming molecules - have been investigated in the quantum regime using cold atom ensembles $[1,2]$ and in the classical regime using optical microdisks [3, 4], microring resonators [5], and excitonpolariton condensates $[6,7]$. Here, we propose such a classical analog simulator for molecular bonding between artificial atoms using extended systems of optically trapped exciton-polariton condensates.

Exciton-polariton condensates in planar microcavities can be confined in etched cavity pillars and mesas, forming a discrete photonic spectrum similar to atomic orbitals [8-10]. When combined, adjacent micropillars become coupled due to the overlapping orbitals forming new macroscopic states $[6,11,12]$. Exciton-polaritons may also be confined optically, by using non-resonant lasers which excite a background exciton gas that repulsively interacts with polaritons [13, 14]. Stimulated bosonic scattering from the surrounding exciton gas then provides gain to the confined condensate, compensating for photonic losses due to the finite microcavity quality factor. In optical traps, polariton condensates demonstrate rich phenomenology, including spin bifurcations [15], quantum chaos [16], vortices [17, 18], spin hysteresis [19], and multilevel synchronization between traps [20].

The advantage of using polariton condensates as macroscopic wavefunction emulators is the easy readout of the polariton state through the emitted cavity light. It permits both integrated and time-resolved measurement of the energy-resolved real-space and momentum-space condensate density, phase, spin (polarization), and correlations between selected condensates [21]. In contrast, high-resolution time-of-flight experiments using ultracold atoms can only indirectly measure correlations and the phase of the atomic ensembles by associating the wave function phase with its momentum density distribution nodal lines (i.e., boundaries of $\pi$ phase slips) [1].

Of interest, compared to other methods of trapping polariton condensates $[6,22]$, optical traps result in stable occupation of the excited states [20, 23-28] due to the optical gain provided by the laser. That is, more energetic states penetrate further into the laser-induced potential, experiencing enhanced scattering into the condensate, and remain indefinitely stable as long as the laser is present. This is in contrast to equilibrium condensates which instead relax quickly to the ground state. Moreover, optically induced potentials benefit from being completely reconfigurable by simply reprogramming the shape of the external nonresonant laser light using liquid crystal spatial light modulators, avoiding the costs of fabricating new patterned cavities from scratch. These two properties of optical traps, stable condensation into exited orbitals and flexibility in potential engineering, underpin their appropriateness for possible analog simulation of molecular bonding.

Today, coupled polariton condensates have been proposed to simulate the energy minima of the XY spin system [29], and recent works have started to address their potential to simulate molecular bonding [30]. There, each condensate is cylindrically symmetric and radially emits polaritons equally in all directions. Thus, the coupling strength between adjacent condensates depends only on the spatial separation distance and occupation difference between condensates. However, if individual condensates occupy more complex orbitals, the in-plane emission of polaritons from the condensate becomes anisotropic, and the coupling strength becomes dependent on the relative angle between neighbors. Anisotropic dissipative coupling and symmetry breaking stemming from it have not 
been accounted for in open nonequilibrium condensates, in contrast to spontaneous symmetry breaking resulting from nonlinear dissipative terms $[15,31,32]$.

In this work we show that anisotropic dissipative coupling of optically trapped and spatially separated polariton condensates demonstrates unique features even in the linear regime close to the condensation threshold. Namely, it results in self-ordering of the macroscopic polariton orbitals orientation maximizing the system gain. This effect is present in the coupling of degenerate orbitals in adjacent optically induced traps. In particular, we consider the first excited doublet of optically confined polariton modes, which are similar to atomic $p$-orbitals. Coupled trapped condensates demonstrate mutual alignment of dipole spatial wavefunction profiles, similarly to $\pi$ and $\sigma$ molecular bond types. The particular type of bond alignment due to the dissipative mechanism depends on the separation distance and the angle between traps. This observation paves the way towards optically controlled emulation of coupling and hybridization of electronic orbitals in molecules.

We first develop the complex $\delta$ shell potential model of a single trap and compute its eigenstates in Sec. II. Treating coupled traps within the perturbation theory, we then compute the spectrum of synchronized states in Sec. III. We calculate the dependence of the interaction strength on the distance between the traps and identify four possible types of coupling and alignment of dipole $p$-state condensates in adjacent traps in Secs. III A and III B, respectively. The polariton spectrum and condensation in the presence of the considered interaction are analyzed in the cases of two traps in Sec. IV. Polariton condensation into aligned dipole states is further confirmed with numerical simulation of the complex Gross-Pitaevskii equation.

\section{SINGLE TRAP}

The dynamics of polariton condensates interacting with spatially inhomogeneous exciton reservoirs is governed by the generalized (driven-dissipative) GrossPitaevskii equation, coupled to the semiclassical rate equation for the reservoir density [33]:

$$
\begin{aligned}
i \hbar \frac{\partial \Psi}{\partial t} & =\left[-\frac{\hbar^{2} \Delta}{2 m^{*}}+\frac{\alpha+i \beta}{2} N+\alpha_{1}|\Psi|^{2}-i \hbar \frac{\Gamma}{2}\right] \Psi, \\
\frac{\partial N}{\partial t} & =P(r)-\left(\beta|\Psi|^{2}+\gamma\right) N .
\end{aligned}
$$

Here, $\Delta$ denotes the two-dimensional Laplacian operator, $\Psi$ and $N$ are the condensate wavefunction and the reservoir density, $m^{*}$ is the polariton effective mass, $\alpha$ and $\alpha_{1}$ are the interaction constants describing the polariton repulsion off the exciton density and polaritonpolariton repulsion, $\beta$ governs the stimulated scattering from the reservoir into the condensate, $\Gamma$ and $\gamma$ are the polariton and exciton decay rates, and $P(r)$ is the cylin- drically symmetric reservoir pumping rate. We will focus on an annular-shaped laser pumping profile, similar to those used in $[13,14,20,23,25-27,34]$. The laser pump is approximated with a $\delta$ shell ring shape $P(r)=P_{0} \delta(r-R) / R$, where $r=\sqrt{x^{2}+y^{2}}$ is the radial coordinate, $R$ is the trap radius, and $P_{0}$ represents the pumping power.

Close to the condensation threshold (i.e., normal-tocondensed-state phase transition) one may put $|\Psi|^{2} \simeq 0$, neglecting polariton-polariton interactions and reservoir depletion in Eqs. (1) and (2). This approximation allows establishing the hierarchy of the fastest exponential decay (or growth) rates of polariton modes in the system. At threshold, scattering from the reservoir starts exceeding the polariton decay rate, corresponding to a mode with the fastest population growth rate. At higher laser power, beyond the condensation threshold, the condensate state is typically governed by this fastest-growing mode which saturates the reservoir and stabilizes. Polariton interactions, however, can destabilize this state when the mean field energy becomes comparable with other characteristic energy scales [35]. We will not consider this regime and focus instead on the hierarchy of the fastest-growing modes around the threshold.

The solutions corresponding to the linear limit of Eq. (1) are stationary, allowing us to put the left-hand part of Eq. (2) to zero and express the reservoir density as $N=P(r) / \gamma$. The reservoir effect on the condensate then reduces to a complex potential given by

$$
V(r)=V_{0} \frac{\delta(r-R)}{R},
$$

where the potential strength $V_{0}=(\alpha+i \beta) P_{0} /(2 \gamma)$. The generalized Gross-Pitaevskii equation then becomes a dissipative (non-Hermitian) Schrödinger equation for the polariton wave function, which in polar coordinates reads:

$$
\left[-\frac{\hbar^{2}}{2 m^{*}}\left(\frac{1}{r} \frac{\partial}{\partial r} r \frac{\partial}{\partial r}+\frac{1}{r^{2}} \frac{\partial}{\partial \varphi^{2}}\right)+V(r)-i \hbar \frac{\Gamma}{2}\right] \Psi=E \Psi,
$$

where $E$ is the complex valued eigenenergy.

Rotational symmetry of the problem allows us to separate the angular and radial coordinates and express the wavefunction in the form $\Psi=\psi(r) \Phi(\varphi)$, where $\Phi(\varphi)=e^{i m \varphi}$. Substituting into Eq. (4) results in an equation for the radial part:

$$
\left[-\frac{\hbar^{2}}{2 m^{*}}\left(\frac{1}{r} \frac{d}{d r} r \frac{d}{d r}-\frac{m^{2}}{r^{2}}\right)+V(r)-E-i \hbar \frac{\Gamma}{2}\right] \psi=0 .
$$

As we are considering the $\delta$ function potential, inside and outside the trap the equation will take the form

$$
r^{2} \frac{d^{2} \psi}{d r^{2}}+r \frac{d \psi}{d r}+\left[2 m^{*}\left(E+i \hbar \frac{\Gamma}{2}\right) \frac{r^{2}}{\hbar^{2}}-m^{2}\right] \psi=0 .
$$

With the substitution $\rho(r)=\sqrt{2 m^{*}(E+i \hbar \Gamma / 2)} r / \hbar$ (the square root corresponds to the principle value) the equa- 
tion turns into the canonical Bessel equation. In the region $r<R$ the wave function then reads $\psi=A J_{m}(\rho)$, where $J_{m}(\rho)$ is the Bessel function of the first kind and we have taken into account the divergence of the Bessel function of the second kind $Y_{m}(\rho)$ at the origin. For $r>R$ the solution can be expressed as $\psi=B H_{m}^{(1)}(\rho)$, where the Hankel function of the first kind is chosen to converge at $r \rightarrow \infty$, keeping in mind that $\operatorname{Im}\{E+i \Gamma / 2\}>0$ and $\operatorname{Re}\{E+i \Gamma / 2\}>0$. In order to match the solutions at the point $r=R$ one needs to satisfy

$$
A J_{m}\left(\rho_{R}\right)=B H_{m}^{(1)}\left(\rho_{R}\right)
$$

where $\rho_{R}=\rho(R)$, and then should integrate Eq. (5) over the small region $r \in\{R-\delta R, R+\delta R\}$, which results in the following condition:

$$
\left.\frac{d \psi}{d r}\right|_{R-\delta R} ^{R+\delta R}=\frac{c}{R} \psi(R)
$$

where $c=2 m^{*} V_{0} / \hbar^{2}$. One can derive, for $\delta R \rightarrow 0$,

$$
\begin{aligned}
& \left.\frac{d \psi}{d r}\right|_{R-\delta R}=\frac{A \rho_{R}}{R}\left(-J_{m+1}\left(\rho_{R}\right)+\frac{m}{\rho_{R}} J_{m}\left(\rho_{R}\right)\right), \\
& \left.\frac{d \psi}{d r}\right|_{R+\delta R}=\frac{B \rho_{R}}{R}\left(-H_{m+1}^{(1)}\left(\rho_{R}\right)+\frac{m}{\rho_{R}} H_{m}^{(1)}\left(\rho_{R}\right)\right) .
\end{aligned}
$$

Equations (9) and (10) yield the trap's resonance condition, given by the transcendental complex equation

$$
\frac{J_{m}\left(\rho_{R}\right)\left(m H_{m}^{(1)}\left(\rho_{R}\right)-\rho_{R} H_{m+1}^{(1)}\left(\rho_{R}\right)\right)}{H_{m}^{(1)}\left(\rho_{R}\right)\left[m J_{m}\left(\rho_{R}\right)-\rho_{R} J_{m+1}\left(\rho_{R}\right)+c J_{m}\left(\rho_{R}\right)\right]}=1 .
$$

The condensation threshold condition can be defined as the moment when all eigenvalues satisfy $\operatorname{Im}\{E\}<0$ (lossy modes) except for one which satisfies $\operatorname{Im}\{E\}=0$. Any state defined by its angular and radial quantum numbers $m$ and $n$, respectively, can then be associated with the so-called threshold pump power $P_{t}^{n, m}$ when the above condition is satisfied. This quantity may be nondimensionalized for convenience:

$$
p_{t}^{n, m}=\frac{(\alpha+i \beta) m^{*}}{\gamma \hbar^{2}} P_{t}^{n, m} .
$$

The dimensionless trap radius, in turn, reads $\rho_{0}=$ $R \sqrt{m^{*} \Gamma / \hbar}$. We focus on solutions of the resonance condition (11) satisfying $\operatorname{Im}\{E\}=0$. Physically, these solutions would appear first in the experimentally measured cavity photoluminescence intensity with increasing pump power. We are interested only in the states $n=0$ as they possess the lowest threshold powers among the states with a given angular number $m$ [36]. This is in agreement with previous experimental observations [23, 25, 27].

The solutions are summarized in Fig. 1(a). For small traps, as expected, the condensate forms at the state $m=0$. However, for larger traps the ground state (a)

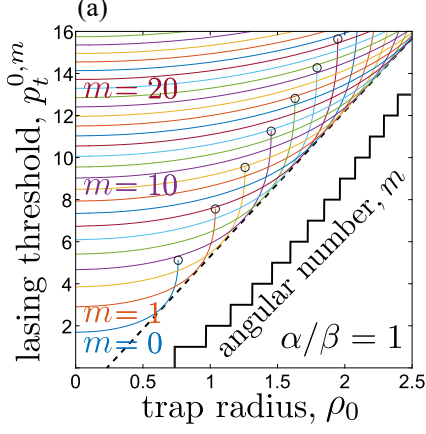

(b)
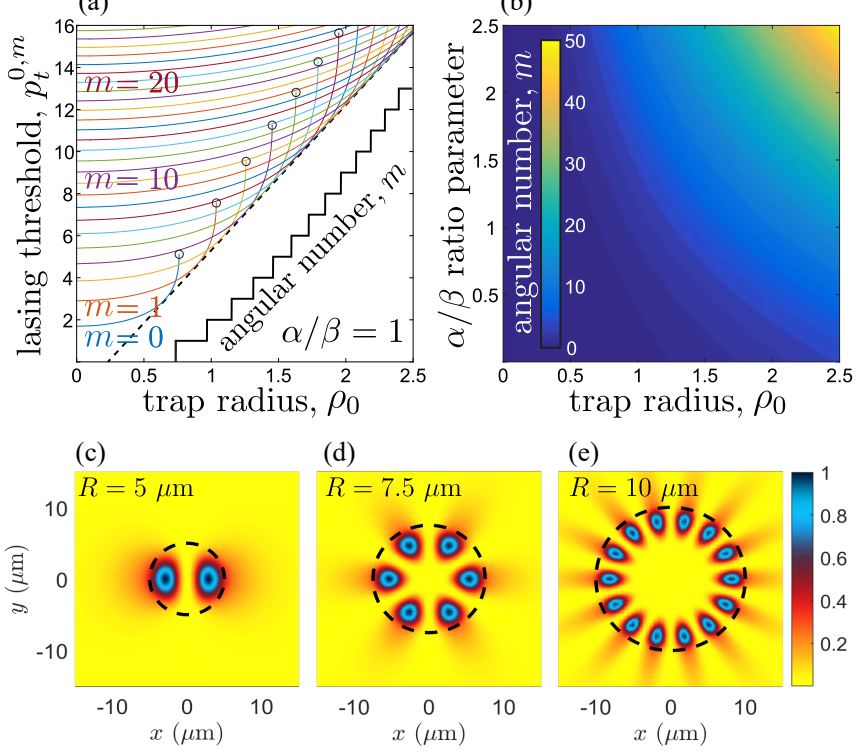

(d)

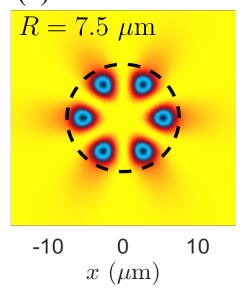

(e)

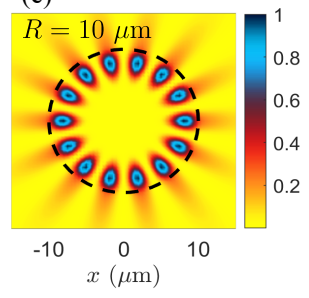

FIG. 1. (a) Condensation (lasing) threshold as a function of the trap radius for $\alpha / \beta=1$. Each state is sustainable only below a certain critical radius (black circles). The threshold state angular number $m$ switches in a ladder like dependence with trap radius (black solid line). The minimal threshold for wide traps $(\rho \gg 1)$ has a linear characteristic dependence on $\rho$, shown with a dashed line. (b) Angular number of the state at which condensation occurs. (c)-(e) Simulated lowest threshold steady-state solutions $|\Psi(\mathbf{r})|^{2}$ of Eqs. (1) and (2) for a single trap of different radii $R=5,7.5,10 \mu \mathrm{m}$, obtained for powers $P_{0} / R=25,40,55 \mu \mathrm{m}^{-2} \mathrm{ps}^{-1}$, respectively. The trap ridge (i.e., circle of radius $R$ ) is given by the dashed black line.

surpasses the first excited doublet $m= \pm 1$ in threshold pumping power. The consequent cascade of successive switching of states with increasing angular numbers results in a dependence of the minimal threshold, resembling a linear one at large scale [see dashed line in Fig. 1(a)]. In Fig. 1(b) we plot an exhaustive phase diagram of the lowest threshold angular number as a function of two dimensionless parameters, the interaction parameter ratio $\alpha / \beta$ and the trap radius $\rho_{0}$. In Figs. 1(c)1 (e) we show real-space densities $|\Psi(\mathbf{r})|^{2}$ of the lowest threshold stationary solutions from Gross-Pitaevskii simulations [Eqs. (1) and (2)] using a pump profile corresponding to current experimental capabilities,

$$
P(r)=\frac{P_{0}}{R} \frac{L_{0}^{4}}{\left(r^{2}-R^{2}\right)^{2}+L_{0}^{4}} .
$$

Here, $L_{0}$ denotes the finite thickness of the reservoirinduced trap walls. The initial condition of the simulations in Figs. 1(c)-1(e) was chosen to be $\Psi(t=0) \propto$ $\cos (m \varphi)$, where the lowest-threshold solution was determined for each $R$ by varying $P_{0}$ slowly across different values of $m$. In all simulations throughout the paper we have chosen parameters similar to those in previous

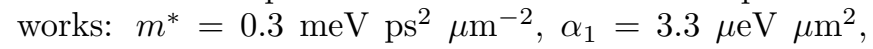


$\alpha=2 \alpha_{1}, \beta=1.4 \alpha_{1}, \gamma=\Gamma=0.2 \mathrm{ps}^{-1}, L_{0}=3 \mu \mathrm{m}$. In Figs. 2, 4, and 5 below we used $P_{0} / R=25 \mu \mathrm{m}^{-2}$ $\mathrm{ps}^{-1}$. In Fig. 4 for the $L_{0}=2 \mu \mathrm{m}$ results we change only $\beta=2.8 \alpha_{1}$.

\section{COUPLING BETWEEN TRAPS}

We will now formulate our theory describing the anisotropic nature of the coupling between spatially separated optically trapped polariton condensates, each populating an excited state in its respective trap. It should be underlined that the mechanism of coupling and synchronization in optically trapped polariton condensates [34] can be drastically different from its counterpart in microcavity pillar structures [6] or in planar microcavities in the presence of potential disorder [37]. In the latter case, polariton tunneling between overlapping orbitals is responsible for their coupling (i.e., evanescent coupling), realizing the familiar bosonic Josephson junction. In the former case, however, condensates excited by the nonresonant laser beams are not separated by a potential barrier save the one which traps themselves. This means that polaritons which escape their optical traps convert trap energy into kinetic energy and propagate across the potential free region between the traps. Such condensates are referred to as ballistic condensates and have been found to stabilize and phase lock over long separation distances by using either Gaussian excitation beams [38, 39] or optical traps like those considered here [34]. Dissipative coupling then refers to the interference (i.e., overlap) of the two condensates in the optical gain region - corresponding to $\operatorname{Im}[V(r)]$ - that changes the imaginary part of the system complex energies. In other words, it is the imaginary (non-Hermitian) part from the overlap integrals between two wave functions and the potential with which they interfere $\langle\Psi|V(\mathbf{r})| \Psi\rangle$. Dissipative coupling is responsible for emergence of the weak lasing regime $[32,35]$, limit cycles [39, 40], and chaos [41]. As discussed in the context of Eq. (12), the imaginary part of a mode's complex energy corresponds to its gain (like optical gain in lasers), and the mode with the highest imaginary part will be occupied by the condensate.

Furthermore, depending on the shape of the laser, ballistic propagation of polaritons from the condensate can become pronounced [see, e.g., concentric rings in Fig. 2(a)], which leads to stronger matter-wave interference between neighbors. The range of this propagation is limited only by the polariton attenuation length and has been reported to synchronize condensates over 100 $\mu \mathrm{m}$ [39], about 50 times longer than the condensate full width at half maximum.

Assuming that the threshold is reached for the first excited mode doublet $m= \pm 1$, as shown schematically in Fig. 2, the general form of the condensate wave function outside the trap reads

$$
\Psi=\left(c_{+} e^{i \varphi}+c_{-} e^{-i \varphi}\right) H_{1}^{(1)}(\rho) .
$$
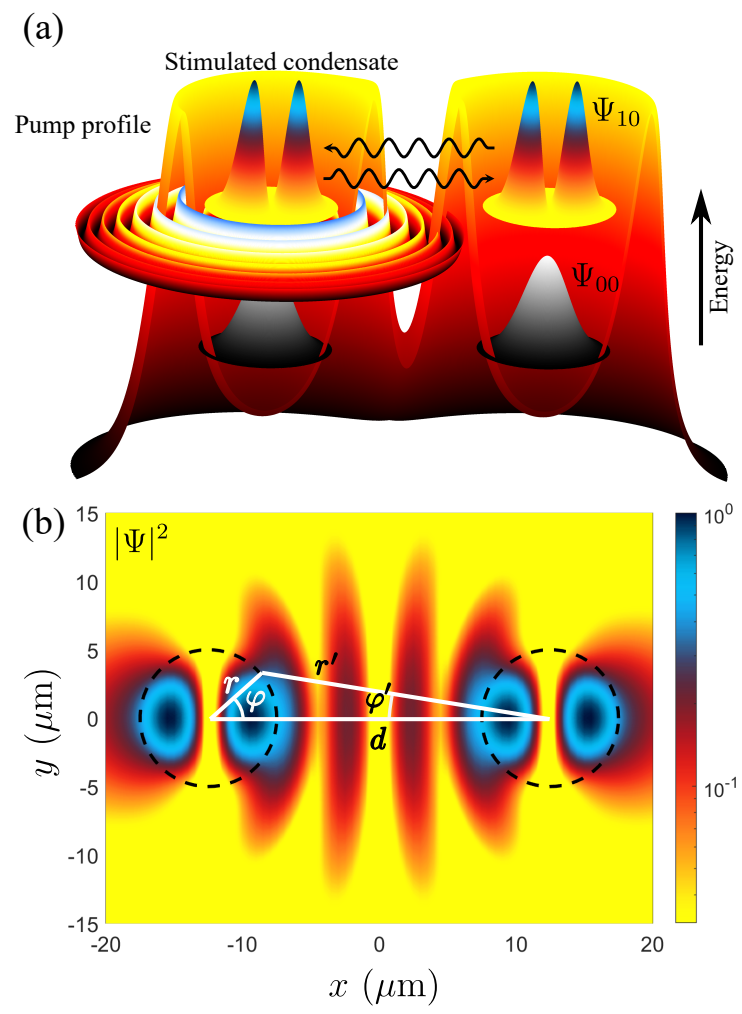

FIG. 2. (a) Schematic of two circular pump-induced potential traps containing the first two Laguerre-Gaussian modes, the ground state $\Psi_{00}$, and the degenerate first excited state $\Psi_{10}$. Finite tunneling probability causes polariton waves to leak radially away from the trap and interfere with any neighboring condensates. (b) Steady-state simulation for $d=25 \mu \mathrm{m}$ showing $|\Psi(\mathbf{r})|^{2}$ with clear interference fringes between two phase locked dipole-shaped condensates analogous to the chemical $\sigma$-bonding configuration. Notation in the text is overlaid for clarity. Black dashed circles denote the ridges of the potential traps.

It is worth noting that these states have been realized several times in experiment [17, 18, 26, 42, 43] but have lacked the theory explaining their coupling in extended systems. Here, the two complex coefficients $c_{ \pm}$are related to the pseudovector $\mathbf{s}=\left(s_{x}, s_{y}, s_{z}\right)^{T}$, which defines its state up to a common gauge-invariant phase:

$$
\begin{aligned}
s_{x}+i s_{y} & =c_{+}^{*} c_{-}, \\
s_{z} & =\left(\left|c_{+}\right|^{2}-\left|c_{-}\right|^{2}\right) / 2 .
\end{aligned}
$$

In this notation, $s_{z}= \pm 1$ corresponds to the two opposite circulating vortex states whereas $s_{x}= \pm 1$ and $s_{y}= \pm 1$ correspond to a dipole state orientated horizontally, vertically, diagonally, and antidiagonally respectively, analogous to the Stokes components of light.

For a fixed value of the condensate energy $E$ we may introduce the characteristic wavevector of the condensate outside the trap $k=\sqrt{2 m^{*}(E+i \hbar \Gamma / 2)} / \hbar$. A pair of coupled condensates in optically induced traps, separated by the distance $d>2 R$, measured from the center of each trap, are then given by a common wave function defined 
by the four coefficients $c_{1 \pm}$, and $c_{2 \pm}$ :

$$
\begin{array}{r}
\Psi(r, \varphi)=\left(c_{1+} e^{i \varphi}+c_{1-} e^{-i \varphi}\right) H_{1}^{(1)}(k r) \\
+\left(c_{2+} e^{i\left(\pi-\varphi^{\prime}\right)}+c_{2-} e^{-i\left(\pi-\varphi^{\prime}\right)}\right) H_{1}^{(1)}\left(k r^{\prime}\right),
\end{array}
$$

where

$$
\begin{aligned}
& r^{\prime}=\sqrt{r^{2}+d^{2}-2 r d \cos (\varphi)}, \\
& \sin \left(\varphi^{\prime}\right)=\sin (\varphi) r / r^{\prime}
\end{aligned}
$$

from the law of cosines and sines, respectively.

In order to investigate the growth rates of coupled states stemming from their interference we directly calculate the overlap integrals between the two wavefunctions and their respective potentials,

$$
I=I^{(1)}+I^{(2)}=\int \Psi^{*}\left[\delta(r-R)+\delta\left(r^{\prime}-R\right)\right] \Psi d \mathbf{r}
$$

The common (superposed) wavefunction overlap with the potential is composed of two terms, corresponding to the two excitonic traps. Let us find them separately and begin with the overlap with the first trap $I^{(1)}=\int_{0}^{2 \pi}|\Psi(R, \varphi)|^{2} d \varphi$, which, in turn, may be decomposed into the sum of overlaps of both individual condensates and the cross term:

$$
\begin{aligned}
I^{(1)} & =I_{0}^{(1)}\left(c_{1+}, c_{1-}\right)+I_{\kappa}^{(1)}\left(c_{2+}, c_{2-}\right) \\
& +I_{\lambda}^{(1)}\left(c_{1+}, c_{1-}, c_{2+}, c_{2-}\right) .
\end{aligned}
$$

The condensate overlap with its own trap reads simply $I_{0}^{(1)}=4 \pi s_{1}\left|H_{1}^{(1)}(k R)\right|^{2}$, where $s_{1,2}=\left|\mathbf{s}_{1,2}\right|$ are the pseudovector amplitudes of each condensate. We note that in standard tight-binding techniques the overlap integrals correspond to constant matrix terms $\langle\Psi|V| \Psi\rangle$ which lead to an eigenvalue problem whose solutions are the new energies and states of the coupled system. However, the ballistic nature of the polaritons is an interference problem, meaning that all integrals depend on the polariton outflow wave vector $k$, which is defined by the energy of the system. For this reason, the new energies of the system cannot be calculated from an eigenvalue problem unless one considers the interaction as a perturbation to the isolated condensate energy [see Eq. (11)]. We will consider this perturbative tight-binding case in Sec. IV.

The interference term reads

$$
\begin{aligned}
I_{\lambda}^{(1)}= & -\int_{0}^{2 \pi}\left(c_{1+} e^{i \varphi}+c_{1-} e^{-i \varphi}\right)\left(c_{2+}^{*} e^{i \varphi^{\prime}}+c_{2-}^{*} e^{-i \varphi^{\prime}}\right) \\
& \times H_{1}^{(1)}(k R) H_{1}^{(1)}\left(k r^{\prime}\right)^{*} d \varphi+\text { c.c. }
\end{aligned}
$$

The second integral due to the second condensate overlap with the first trap reads

$$
I_{\kappa}^{(1)}=\int_{0}^{2 \pi}\left|c_{2+} e^{-i \varphi^{\prime}}+c_{2-} e^{i \varphi^{\prime}}\right|^{2}\left|H_{1}^{(1)}\left(k r^{\prime}\right)\right|^{2} d \varphi .
$$

With increasing distance between the traps $d / R$ the hierarchy of the terms $I_{0}^{(1)} \gg I_{\lambda}^{(1)} \gg I_{\kappa}^{(1)}$ quickly establishes due to exponential decay of the Hankel function.
This allows us to neglect the last term $I_{\kappa}^{(1)}$ and treat the effect of interference $I_{\lambda}^{(1)}$ as a perturbation to the first order with respect to decoupled condensates. The same argument obviously applies to the interference of the condensates at the second trap, with the corresponding integrals being $I_{0}^{(2)}=4 \pi s_{2}\left|H_{1}^{(1)}(k R)\right|^{2}$ and

$$
\begin{aligned}
I_{\lambda}^{(2)}= & \int_{0}^{2 \pi}\left(c_{2+} e^{i \varphi}+c_{2-} e^{-i \varphi}\right)\left(c_{1+}^{*} e^{-i \varphi^{\prime}}+c_{1-}^{*} e^{i \varphi^{\prime}}\right) \\
& \times H_{1}^{(1)}(k R) H_{1}^{(1)}\left(k r^{\prime}\right)^{*} d \varphi+\text { c.c. }
\end{aligned}
$$

where $r^{\prime}=\sqrt{d^{2}+R^{2}+2 d R \cos (\varphi)}$ is slightly redefined compared to the first trap case.

\section{A. Long-distance limit}

Expanding Eqs. (17) and (18) in $R / d \rightarrow 0$, we obtain in the integration range

$$
\varphi^{\prime} \approx \sin (\varphi) \frac{R}{d} \ll 1, \quad r^{\prime} \approx d\left(1-\frac{R}{d} \cos (\varphi)\right) .
$$

In the main order in $R / d$ the interference integral (21) thus reads

$$
\begin{array}{r}
I_{\lambda}^{(1)}=-\int_{0}^{2 \pi}\left(c_{1+} e^{i \varphi}+c_{1-} e^{-i \varphi}\right) \\
\times\left[c_{2+}^{*}\left(1+i \frac{R}{d} \sin (\varphi)\right)+c_{2-}^{*}\left(1-i \frac{R}{d} \sin (\varphi)\right)\right] \\
\times H_{1}^{(1)}(k R) H_{1}^{(1)}[k d-k R \cos (\varphi)]^{*} d \varphi+\text { c.c. }
\end{array}
$$

It is important to note here that $|k| \sim R^{-1}$ since the characteristic wave vector, corresponding to the highest state confined in a trap, is given by the inverse trap size. We may thus asymptotically expand the Hankel function in Eq. (25):

$$
\begin{array}{r}
H_{1}^{(1)}[k d-k R \cos (\varphi)] \approx \frac{(1+i)}{\sqrt{\pi k d}}\left[1+\frac{R}{2 d} \cos (\varphi)\right] \\
\times \exp (i k d) \exp [-i k R \cos (\varphi)] .
\end{array}
$$

Plugging (26) into (25) and employing the integral expression for the Bessel function $2 \pi i J_{1}(\rho)=$ $\int_{0}^{2 \pi} \cos (\varphi) \exp [i \rho \cos (\varphi)] d \varphi$, we may compute the interference integral (25) to the leading order in $R / d$ :

$$
\begin{aligned}
& I_{\lambda}^{(1)}=- 2 \pi i H_{1}^{(1)}(k R)\left(H_{1}^{(1)}(k d) J_{1}(k R)\right)^{*} \\
& \times\left(c_{1+}+c_{1-}\right)\left(c_{2+}+c_{2-}\right)^{*}+\text { c.c. }
\end{aligned}
$$

The analogous interference integral for the first condensate overlap with the second trap reads

$$
\begin{aligned}
& I_{\lambda}^{(2)}=- 2 \pi i H_{1}^{(1)}(k R)\left(H_{1}^{(1)}(k d) J_{1}(k R)\right)^{*} \\
& \times\left(c_{1+}+c_{1-}\right)^{*}\left(c_{2+}+c_{2-}\right)+\text { c.c. }
\end{aligned}
$$


(a)

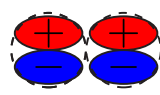

A

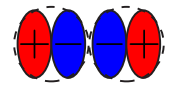

$\mathrm{B}$

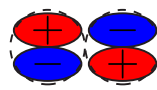

C

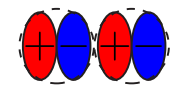

$\mathrm{D}$

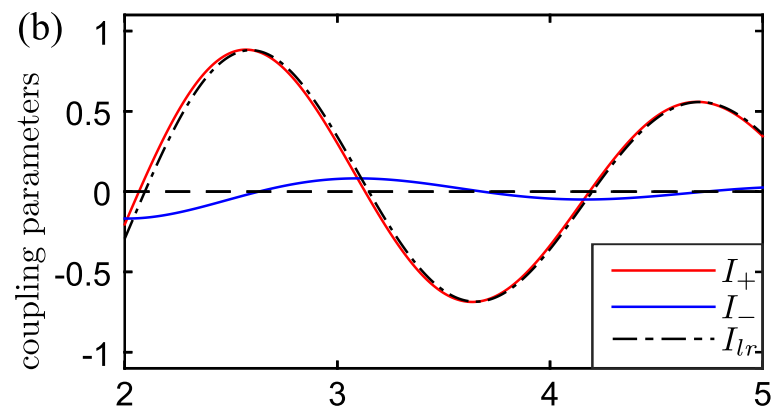

relative distance between traps $d / R$

(c)

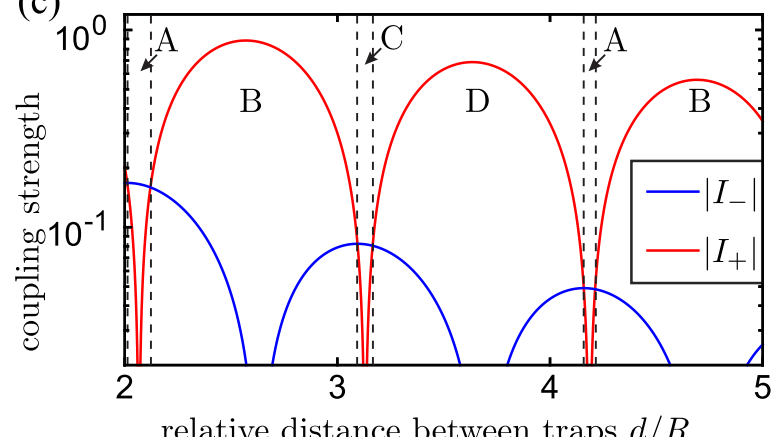

FIG. 3. (a) Illustration of the four possible coupling types. Regimes $\mathrm{A}$ and $\mathrm{C}$ share similarity with $\pi$ bonding, while $\mathrm{B}$ and $\mathrm{D}$ are similar to $\sigma$ bonds. (b) Coupling parameters for $|m|=1$ condensate interference for the experimentally realistic parameter value $k R=3+0.075 i$. (c) The absolute values of coupling parameters. Switching of the coupling types occurs at the crossing points $\left|I_{+}\right|=\left|I_{-}\right|$.

Therefore, we have, for the net interference contribution to the condensate overlap with the reservoir,

$$
\begin{aligned}
I_{\text {int }}= & I_{\lambda}^{(1)}+I_{\lambda}^{(2)} \\
= & 8 \pi \operatorname{Im}\left\{H_{1}^{(1)}(k R)\left(H_{1}^{(1)}(k d) J_{1}(k R)\right)^{*}\right\} \\
& \times \operatorname{Re}\left\{\left(c_{1+}+c_{1-}\right)\left(c_{2+}+c_{2-}\right)^{*}\right\} .
\end{aligned}
$$

\section{B. General case}

One may show for any distance $d$ between the traps that the overlap integral may be represented in the following form:

$$
\begin{aligned}
I_{\text {int }} & =I_{+} \operatorname{Re}\left\{\left(c_{1+}+c_{1-}\right)\left(c_{2+}+c_{2-}\right)^{*}\right\} \\
& +I_{-} \operatorname{Re}\left\{\left(c_{1+}-c_{1-}\right)\left(c_{2+}-c_{2-}\right)^{*}\right\},
\end{aligned}
$$

where $I_{ \pm}=I_{a} \pm I_{b}$ and

$$
\begin{aligned}
I_{a}= & \int_{0}^{2 \pi}\left[e^{i\left(\varphi_{+}^{\prime}-\varphi\right)} H_{1}^{(1)}(k R)^{*} H_{1}^{(1)}\left(k r_{+}^{\prime}\right)\right. \\
& \left.-e^{i\left(\varphi_{-}^{\prime}+\varphi\right)} H_{1}^{(1)}(k R) H_{1}^{(1)}\left(k r_{-}^{\prime}\right)^{*}\right] d \varphi \\
I_{b}= & \int_{0}^{2 \pi}\left[e^{-i\left(\varphi+\varphi_{+}^{\prime}\right)} H_{1}^{(1)}(k R)^{*} H_{1}^{(1)}\left(k r_{+}^{\prime}\right)\right. \\
& \left.-e^{i\left(\varphi-\varphi_{-}^{\prime}\right)} H_{1}^{(1)}(k R) H_{1}^{(1)}\left(k r_{-}^{\prime}\right)^{*}\right] d \varphi
\end{aligned}
$$

where $r_{ \pm}^{\prime}=\sqrt{d^{2}+R^{2} \pm 2 d R \cos (\varphi)}$ and $\varphi_{ \pm}^{\prime}=$ $\sin ^{-1}\left[\sin (\varphi) R / r_{ \pm}^{\prime}\right]$. Physically, the integrals $I_{ \pm}$quantify the strength of interference coming from dipoles that are aligned longitudinally or transversely to the interference direction (the axis connecting the trap centers). Comparing Eqs. (29) and (30), one may note that for long distances there exists an asymptotic relation:

$I_{+}(d) \approx I_{l r}(d)=8 \pi \operatorname{Im}\left\{H_{1}^{(1)}(k R)\left(H_{1}^{(1)}(k d) J_{1}(k R)\right)^{*}\right\}$,

where $I_{l r}(d)$ is the long-range part of the coupling parameter. The numerically computed values of the interaction parameters $I_{ \pm}$are shown in Fig. 3 for the experimentally realistic value of $k R=3+0.075 i$. The asymptotic equivalence of the computed parameters $I_{+}(d)$ and $I_{l r}(d)$ is illustrated in Fig. 3(b) by the solid red and dot-dashed black lines, respectively. Moreover, in the logarithmic scale [Fig. 3(c)] it is clear that the envelope of $\left|I_{+}(d)\right|$ is exponentially dominant in the limit of long distances. Nevertheless, the sign-changing and oscillating character of its dependence on the distance suggests that $\left|I_{-}\right|$, responsible for the $\pi$ bonding type (regimes $\mathrm{A}$ and $\mathrm{C}$ ), overcomes $\left|I_{+}\right|$in narrow regions of distances.

The net overlap integral may be represented in a more compact form, $I=C^{\dagger} \hat{I} C$, in terms of the state vector $C=\left(c_{1+}, c_{1-}, c_{2+}, c_{2-}\right)^{T}$, where

$$
\hat{I}=\left(\begin{array}{cccc}
0 & 0 & I_{a} & I_{b} e^{2 i \theta} \\
0 & 0 & I_{b} e^{-2 i \theta} & I_{a} \\
I_{a} & I_{b} e^{2 i \theta} & 0 & 0 \\
I_{b} e^{-2 i \theta} & I_{a} & 0 & 0
\end{array}\right) .
$$

Here, we have accounted for an arbitrary angle $\theta$ between the axis, connecting the centers of the two traps, and the axis, from which the angles $\varphi$ and $\varphi^{\prime}$ are measured.

\section{THE CASE OF JUST TWO TRAPS}

The coupling derived in the previous section may be applied to various configurations of traps. The simplest case, illustrating the properties of the $p$-orbital dissipative coupling, is the case of only two traps, similar to the $s$-orbital coupling studied in [34]. We compare our analytical results to simulations of the driven-dissipative Gross-Pitaevskii equation [Eqs. (1) and (2)]. 
In the particular case of only two coupled condensates we will write $c_{1 \pm}=\sqrt{s_{1}} \exp \left[i\left(\Phi_{1} \mp \phi_{1}\right)\right], c_{2 \pm}=$ $\sqrt{s_{2}} \exp \left[i\left(\Phi_{2} \mp \phi_{2}\right)\right]$, where $\Phi_{1,2}$ and $\phi_{1,2}$ define the phase of each condensate. We then get

$$
\begin{aligned}
& \operatorname{Re}\left\{\left(c_{1+}+c_{1-}\right)\left(c_{2+}+c_{2-}\right)^{*}\right\} \\
& =4 \sqrt{s_{1} s_{2}} \cos (\Delta \Phi) \cos \left(\phi_{1}\right) \cos \left(\phi_{2}\right), \\
& \operatorname{Re}\left\{\left(c_{1+}-c_{1-}\right)\left(c_{2+}-c_{2-}\right)^{*}\right\} \\
& =4 \sqrt{s_{1} s_{2}} \cos (\Delta \Phi) \sin \left(\phi_{1}\right) \sin \left(\phi_{2}\right),
\end{aligned}
$$

where $\Delta \Phi=\Phi_{2}-\Phi_{1}$. Substituting Eqs. (35) and (36) into (30), we obtain:

$$
\begin{aligned}
I & =4 \sqrt{s_{1} s_{2}} \cos (\Delta \Phi) \\
& \times\left[I_{+} \cos \left(\phi_{1}\right) \cos \left(\phi_{2}\right)+I_{-} \sin \left(\phi_{1}\right) \sin \left(\phi_{2}\right)\right] .
\end{aligned}
$$

The relative "external" phase difference between the two condensates $\Delta \Phi$, and the "internal" phases $\phi_{1}$ and $\phi_{2}$ are chosen to maximize the value of the overlap integral (37).

We will consider the limiting case of the outflow wave vector $k=k_{0}$ being approximately unaffected by the coupling, where $k_{0}$ is the wave vector corresponding to a single isolated condensate. The problem of identifying the solutions of the system in the linear regime is then analogous to a tight-binding theory where the new Hamiltonian describing the coupled system can be written

$$
\hat{H}=\frac{V_{0} \hat{I}\left(k_{0}\right)}{R} .
$$

Eigenvalues of Eq. (38) are complex since $V_{0}$ is complex, with the largest imaginary part eigenvalue corresponding to the lowest-threshold solution. We can directly infer from the relative strength and signs of the overlap integrals $I_{ \pm}$and the fact that $\operatorname{Im}\left(V_{0}\right)>0$, which molecule configuration [see A-D in Fig. 3(a)] maximizes the gain of the system.

If $\left|I_{+}\right|>\left|I_{-}\right|$, then $\phi_{1}$ and $\phi_{2}$ take the values $\pi n$, corresponding to two dipole states aligned so that all four polariton density peaks lie on the line, connecting the centers of the traps. The relative phase $\Delta \Phi$ which leads to the strongest constructive interference and maximum gain is governed by the sign of $I_{+}$: a negative sign corresponds to a symmetric wave function (B), while positive sign corresponds an antisymmetric wave function (D).

If, on the contrary, $\left|I_{+}\right|<\left|I_{-}\right|$, then $\phi_{1}$ and $\phi_{2}$ take the values $\pi(n+1 / 2)$, corresponding to both dipoles orientated transversely to the axis connecting the traps. Positive values of $I_{-}$again correspond to a symmetric wave function (A), while negative values correspond to an antisymmetric wave function $(\mathrm{C})$.

We can notice that the condensate $\sigma$ bonds [configurations B and D in Fig. 3(a)] possess the lowest threshold over a much wider range compared to the $\pi$ bonds [configurations $\mathrm{A}$ and $\mathrm{C}$ in Fig. 3(a)]. In fact, this is analogous to molecular orbital theory where $\sigma$ bonds form the
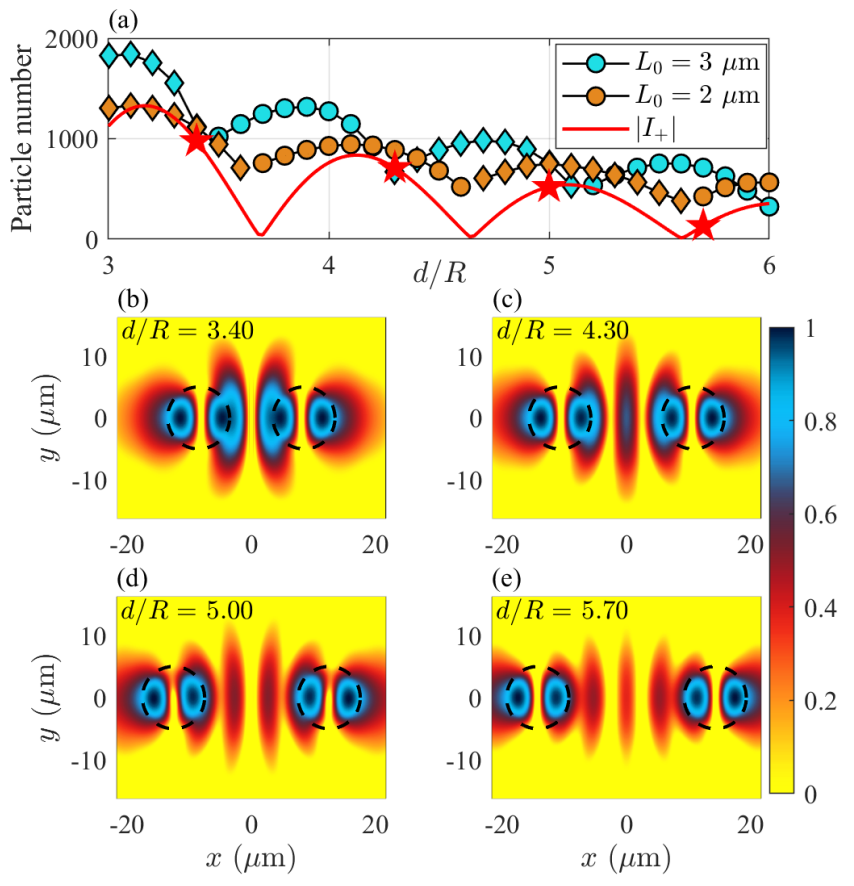

FIG. 4. Distance dependence of the stable steady-state solutions from simulation of the dissipative Gross-Pitaevskii equation for two traps of $R=5 \mu \mathrm{m}$. The observed states correspond to configurations B and D in Fig. 3(a) and are obtained from stochastic initial conditions. (a) Particle number of the two coupled condensate system for two different values of $L_{0}$. Circles correspond to state $\mathrm{B}$, and diamonds to state D. The oscillating number of particles for $L_{0}=2 \mu \mathrm{m}$ is well matched by our interference expression $\left|I_{+}\right|$(scaled) depicting the gain of the $\sigma$ bonds (red solid line). Here, we use $k R=3.96+i 0.41$, estimated from an isolated condensate steady state. For $L_{0}=3 \mu \mathrm{m}$ we observe stronger deviation from the $\delta$ shell model, as expected. (b)-(e) The corresponding normalized condensate density $|\Psi(\mathbf{r})|^{2}$ is shown for the case of $L_{0}=2 \mu \mathrm{m}$ for four different values of $d / R$ (red stars). Black dashed circles denote the ridges of the traps.

strongest covalent bonds, determined by the amount of overlap between the atomic orbitals. For the experimentally relevant set of system parameters the stable existence of only the $\sigma$ configurations (i.e., states B and D) is confirmed through simulation of the driven-dissipative Gross-Pitaevskii equation, where in Fig. 4 we show the resulting condensate steady states from stochastic initial conditions as a function of trap distance. While $\pi$ bonds are predicted to exist in the linear regime at key distances for ideal $\delta$ shell potentials there are several elements that could explain their absence in simulation. First, the simulation operates in the nonlinear regime since the condensate converges to a steady state. Second, the pump profile [see Eq. (13)] has not reached the $\delta$ function limit. Third, the wave vector $k$ entering the overlap integrals [Eqs. (31) and (32)] changes slightly as the coupled system energy changes. 

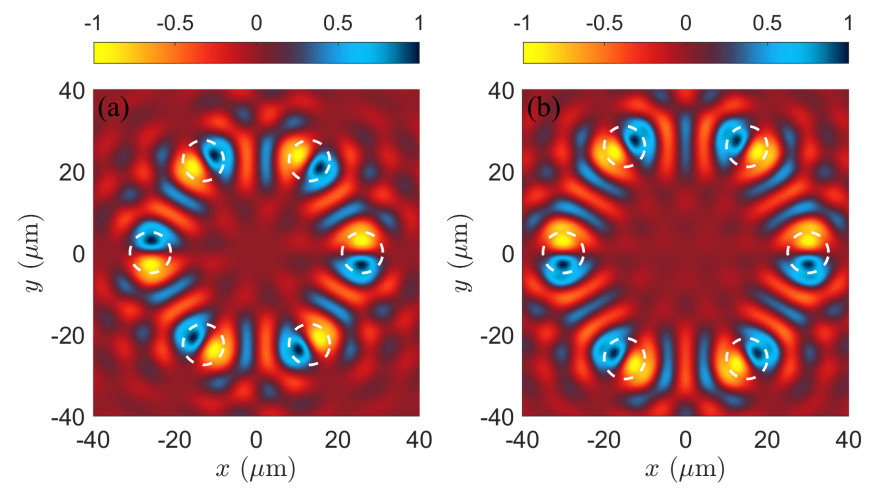

FIG. 5. The two stable $\sigma$ bond configurations $\operatorname{Re}[\Psi(\mathbf{r})]$ (normalized) from the simulation of the dissipative GrossPitaevskii equation for a hexagon of traps of $R=5 \mu \mathrm{m}$ and circumradius $R_{\text {hex }}=26,30 \mu \mathrm{m}$ in (a) and (b) respectively. White dashed circles denote the ridges of the traps.

We will then focus on just the observed $\sigma$ bonds. In Fig. 4(a) we plot the particle number $\int|\Psi|^{2} d \mathbf{r}$, which shows an oscillating character changing from state B (circles) to state D (diamonds) at intervals. This is in good agreement with our derived interference expression $\left|I_{+}\right|$ (red solid curve) from the $\delta$ shell model. When $I_{+}>0$ occurs we obtain state $B$, which maximizes the gain of the system, whereas when $I_{+}<0$ we obtain state $D$ in the same way. As the distance $d$ increases, the particle number reduces as the interference weakens between condensates. To calculated $\left|I_{+}\right|$, we used $\operatorname{Re}[k]=0.66 \mu \mathrm{m}^{-1}$ obtained from the isolated condensate steady state. We then estimated $\operatorname{Im}[k]=\Gamma m^{*} / 2 \hbar \operatorname{Re}[k]$, corresponding to the decaying envelope of the polariton Bessel function away from the trap [44]. The best fit for $\left|I_{+}\right|$was then obtained using $R=6 \mu \mathrm{m}$, which is slightly larger than the value $R=5 \mu \mathrm{m}$ used in simulation. We attribute this deviation to the fact that $L_{0}=2 \mu \mathrm{m}$ has not fully reached the $\delta$ shell limit and that coupling between the condensates changes the system energy, which affects $\operatorname{Re}[k]$. As expected, for $L_{0}=3 \mu \mathrm{m}$, corresponding to a trap with thicker walls, we observe stronger deviation from the $\delta$ shell model. In Figs. 4(b)-4(e) we show the corresponding normalized condensate densities for four distances $d / R$ [red stars in Fig. 4(a)] in the case of $L_{0}=2 \mu \mathrm{m}$.

We additionally show results in Fig. 5 for a hexagon of coupled dipoles emulating a $\sigma$ bonded benzene-type molecule at two different molecule radii, $R_{\text {hex }}=26,30$ $\mu \mathrm{m}$. The $\sigma$ bonded states are dominantly stable, and we expect that such configurations will appear in experiment. To favor the $\pi$ bonding mechanism, one can introduce eccentricity $<1$ into the traps such that mode splitting occurs and the condensate will start favoring $\pi$ bonded states. Indeed, such control over the trap geometry is easily possible in experiment and would allow for much more complex molecule simulation than shown in Fig. 4 and 5.

\section{CONCLUSION}

We have proposed and investigated a system of exciton-polariton condensates for emulation of atomic orbitals and taken steps towards realization of opticalbased simulation of interacting condensates forming twodimensional molecules. We have focused on the first excited $p$-orbital state in a cylindrically symmetric trap, already observed in a number of polariton experiments [17, 18, 20, 25, 43]. From there, we studied the formation of in-phase and anti-phase $\pi$ - and $\sigma$-type bonding configurations dictated by the system optimal gain, or lowestthreshold, condition due to the dissipative nature of the polaritons. Interestingly, we find that the condensate $\sigma$ bonds are dominant, analogous to molecular bonding theory, in which they form the strongest covalent bonds and most stable molecular configuration. Our trap, which mimics the atomic potential, can be engineered through optical means, allowing for reprogrammable parameters of the potential such as its shape and size. The approach is straightforwardly extendable for the creation of lattices of artificial atoms with independent control over individual trap parameters. This allows for the implementation of a number of different geometries such as square, triagonal, honeycomb, and more, which underscores the capabilities of the platform as a potential analog simulator for conjugated systems of $p$-orbitals and possibly more complex molecules. The condensate occupation number scales with the excitation power, which allows control over the influence of on-site particle interactions. The spin degree of freedom of the condensates can also be harnessed by controlling the excitation polarization, which can be used to explore the spinor states of the designed molecules [43].

Although our theoretical analysis has similarities to the gain maximization principle used for the description of dissipative coupling of polariton condensates [31], we emphasize that the addressed linear model does not rely on the gain maximization assumption, which is invalid in the presence of strong nonlinearities, resulting in spontaneous symmetry breaking. Instead, we identify the coupled state characterized by the lowest condensation threshold in the pumping power, describing adiabatically slow condensation in the vicinity of this threshold, where no mode competition is taking place. Although more detailed numerical simulation accounting for nonlinearity in the experimentally relevant range of parameters supports the analytical results, we are leaving the proper analysis of the stability of predicted molecular condensate states out of the scope of this work.

Beyond simulation of electron dynamics in molecules, our study also brings in the possibility to investigate networks of dissipative polariton condensates with a directional (anisotropic) coupling mechanism. While $p$-orbital interactions of polariton condensates are already well established in high-quality patterned microcavities [6, 12], networks of optical traps containing dissipatively coupled excited condensate orbitals have not been considered be- 
fore. Of interest, by designing traps with open space between their centers like shown in Fig. 2(b), as opposed to traps that are tightly packed, one can introduce the effect of a time lag into the condensate coupling due to the finite travel time of particles. Such time delay coupling [39], available in our system, cannot be reproduced in patterned microcavities or cold atoms which rely on evanescent coupling (tunneling).

\section{ACKNOWLEDGMENTS}

H.S. acknowledges the support of the United Kingdom's Engineering and Physical Sciences Research Council (Grant No. EP/M025330/1 on Hybrid Polaritonics), and the European Union's Horizon 2020 program, through a FET Open research and innovation action under the grant agreement No. 899141 (PoLLoC), and the hospitality provided by the University of Iceland, and useful discussions with S. Harrison and P. G. Lagoudakis. A.V.N. acknowledges support from the European Union's Horizon 2020 research and innovation program under Marie Skłodowska-Curie Grant Agreement No. 846353.
[1] D.-S. Lühmann, C. Weitenberg, and K. Sengstock, Emulating molecular orbitals and electronic dynamics with ultracold atoms, Phys. Rev. X 5, 031016 (2015).

[2] J. Argüello-Luengo, A. González-Tudela, T. Shi, P. Zoller, and J. I. Cirac, Analogue quantum chemistry simulation, Nature 574, 215 (2019).

[3] A. Nakagawa, S. Ishii, and T. Baba, Photonic molecule laser composed of gainasp microdisks, Applied Physics Letters 86, 041112 (2005).

[4] G. Fasching, C. Deutsch, A. Benz, A. M. Andrews, P. Klang, R. Zobl, W. Schrenk, G. Strasser, P. Ragulis, V. Tamošiūnas, and K. Unterrainer, Electrically controllable photonic molecule laser, Opt. Express 17, 20321 (2009).

[5] M. Zhang, C. Wang, Y. Hu, A. Shams-Ansari, T. Ren, S. Fan, and M. Lončar, Electronically programmable photonic molecule, Nature Photonics 13, 36 (2019).

[6] M. Galbiati, L. Ferrier, D. D. Solnyshkov, D. Tanese, E. Wertz, A. Amo, M. Abbarchi, P. Senellart, I. Sagnes, A. Lemaître, E. Galopin, G. Malpuech, and J. Bloch, Polariton condensation in photonic molecules, Phys. Rev. Lett. 108, 126403 (2012).

[7] S. Dufferwiel, F. Li, A. A. P. Trichet, L. Giriunas, P. M. Walker, I. Farrer, D. A. Ritchie, J. M. Smith, M. S. Skolnick, and D. N. Krizhanovskii, Tunable polaritonic molecules in an open microcavity system, Applied Physics Letters 107, 201106 (2015).

[8] R. I. Kaitouni, O. El Daïf, A. Baas, M. Richard, T. Paraiso, P. Lugan, T. Guillet, F. Morier-Genoud, J. D. Ganière, J. L. Staehli, V. Savona, and B. Deveaud, Engineering the spatial confinement of exciton polaritons in semiconductors, Phys. Rev. B 74, 155311 (2006).

[9] D. Bajoni, P. Senellart, E. Wertz, I. Sagnes, A. Miard, A. Lemaître, and J. Bloch, Polariton laser using single micropillar GaAs-GaAlAs semiconductor cavities, Phys. Rev. Lett. 100, 047401 (2008).

[10] L. Ferrier, E. Wertz, R. Johne, D. D. Solnyshkov, P. Senellart, I. Sagnes, A. Lemaître, G. Malpuech, and J. Bloch, Interactions in confined polariton condensates, Phys. Rev. Lett. 106, 126401 (2011).

[11] V. G. Sala, D. D. Solnyshkov, I. Carusotto, T. Jacqmin, A. Lemaître, H. Terças, A. Nalitov, M. Abbarchi, E. Galopin, I. Sagnes, J. Bloch, G. Malpuech, and A. Amo, Spin-orbit coupling for photons and polaritons in microstructures, Phys. Rev. X 5, 011034 (2015).
[12] F. Mangussi, M. Milićević, I. Sagnes, L. L. Gratiet, A. Harouri, A. Lemaître, J. Bloch, A. Amo, and G. Usaj, Multi-orbital tight binding model for cavity-polariton lattices, Journal of Physics: Condensed Matter 32, 315402 (2020).

[13] A. Askitopoulos, H. Ohadi, A. V. Kavokin, Z. Hatzopoulos, P. G. Savvidis, and P. G. Lagoudakis, Polariton condensation in an optically induced two-dimensional potential, Phys. Rev. B 88, 041308 (2013).

[14] P. Cristofolini, A. Dreismann, G. Christmann, G. Franchetti, N. G. Berloff, P. Tsotsis, Z. Hatzopoulos, P. G. Savvidis, and J. J. Baumberg, Optical superfluid phase transitions and trapping of polariton condensates, Phys. Rev. Lett. 110, 186403 (2013).

[15] H. Ohadi, A. J. Ramsay, H. Sigurdsson, Y. del ValleInclan Redondo, S. I. Tsintzos, Z. Hatzopoulos, T. C. H. Liew, I. A. Shelykh, Y. G. Rubo, P. G. Savvidis, and J. J. Baumberg, Spin order and phase transitions in chains of polariton condensates, Phys. Rev. Lett. 119, 067401 (2017).

[16] T. Gao, E. Estrecho, K. Y. Bliokh, T. C. H. Liew, M. D. Fraser, S. Brodbeck, M. Kamp, C. Schneider, S. Höfling, Y. Yamamoto, F. Nori, Y. S. Kivshar, A. G. Truscott, R. G. Dall, and E. A. Ostrovskaya, Observation of nonhermitian degeneracies in a chaotic exciton-polariton billiard, Nature 526, 554 (2015).

[17] T. Gao, G. Li, E. Estrecho, T. C. H. Liew, D. ComberTodd, A. Nalitov, M. Steger, K. West, L. Pfeiffer, D. W. Snoke, A. V. Kavokin, A. G. Truscott, and E. A. Ostrovskaya, Chiral modes at exceptional points in excitonpolariton quantum fluids, Phys. Rev. Lett. 120, 065301 (2018).

[18] X. Ma, B. Berger, M. Aßmann, R. Driben, T. Meier, C. Schneider, S. Höfling, and S. Schumacher, Realization of all-optical vortex switching in exciton-polariton condensates, Nature Communications 11, 897 (2020).

[19] L. Pickup, K. Kalinin, A. Askitopoulos, Z. Hatzopoulos, P. G. Savvidis, N. G. Berloff, and P. G. Lagoudakis, Optical bistability under nonresonant excitation in spinor polariton condensates, Phys. Rev. Lett. 120, 225301 (2018).

[20] J. D. Töpfer, H. Sigurdsson, S. Alyatkin, and P. G. Lagoudakis, Lotka-volterra population dynamics in coherent and tunable oscillators of trapped polariton condensates (2020), arXiv:2009.05637 [cond-mat.mes-hall]. 
[21] J. D. Töpfer, I. Chatzopoulos, H. Sigurdsson, T. Cookson, Y. G. Rubo, and P. G. Lagoudakis, Engineering spatial coherence in lattices of polariton condensates, Optica 8, 106 (2021).

[22] M. Maragkou, A. J. D. Grundy, E. Wertz, A. Lemaître, I. Sagnes, P. Senellart, J. Bloch, and P. G. Lagoudakis, Spontaneous nonground state polariton condensation in pillar microcavities, Phys. Rev. B 81, 081307 (2010).

[23] F. Manni, K. G. Lagoudakis, T. C. H. Liew, R. André, and B. Deveaud-Plédran, Spontaneous pattern formation in a polariton condensate, Phys. Rev. Lett. 107, 106401 (2011).

[24] G. Tosi, G. Christmann, N. G. Berloff, P. Tsotsis, T. Gao, Z. Hatzopoulos, P. G. Savvidis, and J. J. Baumberg, Sculpting oscillators with light within a nonlinear quantum fluid, Nature Physics 8, 190 (2012).

[25] A. Dreismann, P. Cristofolini, R. Balili, G. Christmann, F. Pinsker, N. G. Berloff, Z. Hatzopoulos, P. G. Savvidis, and J. J. Baumberg, Coupled counterrotating polariton condensates in optically defined annular potentials, Proceedings of the National Academy of Sciences 111, 8770 (2014).

[26] A. Askitopoulos, T. C. H. Liew, H. Ohadi, Z. Hatzopoulos, P. G. Savvidis, and P. G. Lagoudakis, Robust platform for engineering pure-quantum-state transitions in polariton condensates, Phys. Rev. B 92, 035305 (2015).

[27] Y. Sun, Y. Yoon, S. Khan, L. Ge, M. Steger, L. N. Pfeiffer, K. West, H. E. Türeci, D. W. Snoke, and K. A. Nelson, Stable switching among high-order modes in polariton condensates, Phys. Rev. B 97, 045303 (2018).

[28] C. Xu, B. Zhou, X. Wang, C. Tian, Y. Zhang, H. Dong, G. Wang, and W. Zhou, Dynamics of excited-state condensate for optically confined exciton-polaritons, Opt. Express 27, 24938 (2019).

[29] N. G. Berloff, M. Silva, K. Kalinin, A. Askitopoulos, J. D. Töpfer, P. Cilibrizzi, W. Langbein, and P. G. Lagoudakis, Realizing the classical xy hamiltonian in polariton simulators, Nature Materials 16, 1120 (2017).

[30] A. Johnston, K. P. Kalinin, and N. G. Berloff, Artificial polariton molecules, Phys. Rev. B 103, L060507 (2021).

[31] H. Ohadi, A. Dreismann, Y. G. Rubo, F. Pinsker, Y. del Valle-Inclan Redondo, S. I. Tsintzos, Z. Hatzopoulos, P. G. Savvidis, and J. J. Baumberg, Spontaneous spin bifurcations and ferromagnetic phase transitions in a spinor exciton-polariton condensate, Phys. Rev. X 5, 031002 (2015).

[32] L. Zhang, W. Xie, J. Wang, A. Poddubny, J. Lu, Y. Wang, J. Gu, W. Liu, D. Xu, X. Shen, Y. G. Rubo,
B. L. Altshuler, A. V. Kavokin, and Z. Chen, Weak lasing in one-dimensional polariton superlattices, Proceedings of the National Academy of Sciences 112, E1516 (2015).

[33] M. Wouters and I. Carusotto, Excitations in a nonequilibrium bose-einstein condensate of exciton polaritons, Phys. Rev. Lett. 99, 140402 (2007).

[34] S. L. Harrison, H. Sigurdsson, and P. G. Lagoudakis, Synchronization in optically trapped polariton stuart-landau networks, Phys. Rev. B 101, 155402 (2020).

[35] I. L. Aleiner, B. L. Altshuler, and Y. G. Rubo, Radiative coupling and weak lasing of exciton-polariton condensates, Phys. Rev. B 85, 121301 (2012).

[36] Condensation into the state $n=1$ was observed in Ref. [25] when the pumping had the shape of two concentric rings.

[37] A. Baas, K. G. Lagoudakis, M. Richard, R. André, L. S. Dang, and B. Deveaud-Plédran, Synchronized and desynchronized phases of exciton-polariton condensates in the presence of disorder, Physical Review Letters 100, 2 (2008).

[38] H. Ohadi, R. L. Gregory, T. Freegarde, Y. G. Rubo, A. V. Kavokin, N. G. Berloff, and P. G. Lagoudakis, Nontrivial phase coupling in polariton multiplets, Phys. Rev. X 6, 031032 (2016).

[39] J. D. Töpfer, H. Sigurdsson, L. Pickup, and P. G. Lagoudakis, Time-delay polaritonics, Communications Physics 3, 2 (2020).

[40] A. V. Nalitov, H. Sigurdsson, S. Morina, Y. S. Krivosenko, I. V. Iorsh, Y. G. Rubo, A. V. Kavokin, and I. A. Shelykh, Optically trapped polariton condensates as semiclassical time crystals, Phys. Rev. A 99, 033830 (2019).

[41] R. Ruiz-Sánchez, R. Rechtman, and Y. G. Rubo, Autonomous chaos of exciton-polariton condensates, Phys. Rev. B 101, 155305 (2020).

[42] R. Cerna, D. Sarchi, T. K. Paraïso, G. Nardin, Y. Léger, M. Richard, B. Pietka, O. El Daif, F. Morier-Genoud, V. Savona, M. T. Portella-Oberli, and B. DeveaudPlédran, Coherent optical control of the wave function of zero-dimensional exciton polaritons, Phys. Rev. B 80, 121309 (2009).

[43] A. Askitopoulos, A. V. Nalitov, E. S. Sedov, L. Pickup, E. D. Cherotchenko, Z. Hatzopoulos, P. G. Savvidis, A. V. Kavokin, and P. G. Lagoudakis, All-optical quantum fluid spin beam splitter, Phys. Rev. B 97, 235303 (2018).

[44] M. Wouters, I. Carusotto, and C. Ciuti, Spatial and spectral shape of inhomogeneous nonequilibrium excitonpolariton condensates, Phys. Rev. B 77, 115340 (2008). 